\section{PSICOLOGIA IBEROAMERICANA}

\section{Psicología Iberoamericana}

ISSN: 1405-0943

psicología.iberoamericana@uia.mx

Universidad Iberoamericana, Ciudad de

México

México

Gurrola Peña, Gloria Margarita; Balcázar Nava, Patricia; Bonilla Muñoz, Martha Patricia; Navarrete

Sánchez, Enrique; Farfán García, Ma. Del Carmen; Santos López, Aristeo

Cambios en la Orientación Personal mediante la Intervención Grupal con Alumnos de Bajo

Rendimiento Escolar

Psicología Iberoamericana, vol. 19, núm. 1, enero-junio, 2011, pp. 19-26

Universidad Iberoamericana, Ciudad de México

Distrito Federal, México

Disponible en: http://www.redalyc.org/articulo.oa?id=133920896003

Cómo citar el artículo

- Número completo

- Más información del artículo

- Página de la revista en redalyc.org

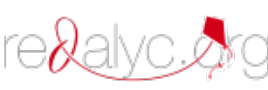

Sistema de Información Científica

Red de Revistas Científicas de América Latina, el Caribe, España y Portugal

Proyecto académico sin fines de lucro, desarrollado bajo la iniciativa de acceso abierto 


\title{
Cambios en la Orientación Personal Mediante la Intervención Grupal con Alumnos de Bajo Rendimiento Escolar
}

\author{
Changes in Personal Orientation of Low Academic Performance Students \\ by Means of Group Intervention
}

\author{
Gloria Margarita Gurrola Peña \\ Patricia Balcázar Nava \\ Martha Patricia Bonilla Muñoz \\ Enrique Navarrete Sánchez \\ Ma. Del Carmen Farfán García \\ Aristeo Santos López* \\ Facultad de Ciencias de la Conducta \\ Universidad Autónoma del Estado de México, México
}

\section{RESUMEN}

El presente estudio tuvo como objetivo determinar el impacto de un programa de atención a alumnos con problemas académicos en sus niveles de autoactualización. Se aplicó el Cuestionario de Orientación Personal diseñado por Shostrom (1963) antes y después de un programa de intervención basado en la búsqueda del autoconocimiento y fortalecimiento de la autoestima a dos grupos de alumnos del sexo masculino a punto de ser dados de baja de una Universidad privada por su bajo rendimiento escolar.

Los resultados indicaron mejoras significativas en seis de las diez escalas clínicas en el primer grupo y en ocho del segundo. De las escalas que mostraron mejoría, las de Espontaneidad, Autoestima y Capacidad de Contacto Íntimo alcanzaron la normalidad estadística, además que 38 de los 40 alumnos participantes lograron superar su estatus académico.

Descriptores: autoactualización, orientación personal, programa de atención, bajo rendimiento escolar, autoestima.

\section{ABSTRACT}

The purpose of this study was to determine the impact of an attention program to students with academic problems in their self-updating levels. It was used the Personal Orientation Questionnaire, designed by Everett Shostrom, before and after of an intervention program based on the search of self-knowledge and self-esteem strengthening in two groups of male students close to be expelled from the University because of their low school performance.

Results show significant improvements in six of ten clinic scales in the first group and in eight in the second group. From the scales which showed improvement, the Spontaneity, Self-Esteem, and Close Contact scales reached the statistic normality, besides that 38 participant students reached to overcome their academic status.

Key words: self-updating, personal orientation, attention program, low school performance, self-esteem

\footnotetext{
* Para correspondencia con: Profesores-investigadores de la Facultad de Ciencias de la Conducta. Universidad Autónoma del Estado de México. Filiberto Gómez s/n. Col. Guadalupe. C.P. 50010.Toluca, Estado de México. Tel: 722278720076.

Gloria Margarita Gurrola Peña: mgurrolaunid@hotmail.com, Patricia Balcázar Nava: pbalcazarnava@hotmail.com, Martha Patricia Bonilla Muñoz: patyb@prodigy.net.mx, Enrique Navarrete Sánchez: navrrete_le@hotmail.com, Ma. Del Carmen Farfán García: mfarfan@itesm.mx, Aristeo Santos López: arisan3@gmail.com.
} 
Fue Maslow (1954) quien dentro de la corriente humanista en psicología introdujo el constructo llamado autoactualización (orientación personal), derivado de sus investigaciones con personas que funcionaban psicológicamente bien, e indicó que una persona que se autoactualiza es una persona muy competente $y$ fuerte a la vez, que tiene conciencia de sus debilidades (Maslow, como se citó en Lafarga y Gómez, 1989). Por su parte, Everett Shostrom (1963) volvió operacional el constructo al indicar que las personas autoactualizantes se experimentan a sí mismas como capaces de enojarse (aceptación de la agresión), de experimentar su debilidad (autoaceptación), de ser amorosas con los demás (capacidad del contacto íntimo) y consigo mismos (autoestima). Además, son personas que aceptan los valores de los demás, son flexibles al aplicar sus propios valores, atienden a sus necesidades y sentimientos, son sensibles a las necesidades de los demás, logran encontrar significado a sus experiencias, llevan a cabo acciones espontáneas, tienen la capacidad de diferenciar polos opuestos y pueden ver las dificultades de la vida como retos.

En este sentido, a pesar de que las personas se caracterizan por tratar de mantener su tendencia actualizante, estudios realizados por Dubois, Felner, Sherman y Bull (citados por Filozof, Albertin, Jones \& Steme, 1998), indican que los eventos negativos de vida, especialmente en los jóvenes, afectan de forma directa al factor actualizante de la autoestima y por ende del autoconcepto. Por otra parte Marsh (1984), exponía que atribuir los éxitos y los fracasos a factores internos controlables (el trabajo y esfuerzo, por ejemplo), está relacionado de manera positiva con el autoconcepto y el rendimiento académico. Adicionalmente, se ha encontrado también que para los adolescentes la mayor fuente de estrés negativo proviene de experiencias problemáticas en las esferas social y académica (Dubois, 1992).

Sin poder determinar si los mismos son causa o efecto de los problemas escolares se ha encontrado que los alumnos con bajo rendimiento escolar muestran baja autoestima y un autoconcepto negativo (Hay, Ashman \& Van Kraayenoord, 1998; Hartocollis, 1999). De esta manera, por ser la autoestima un factor importante en la tendencia actualizante y por lo tanto, en el ajuste psicológico del adolescente (Pope, 1988), en la confianza en sí mismo y en las habilidades en general (Dumont \& Provost, 1999), a la vez que es un buen predictor de menos problemas emocionales y conductuales, así como de altos niveles de desarrollo académico (Dubois, Bull, Sherman \& Roberts, 1998) se justifica el uso de programas de apoyo centrados en el trabajo personal con los alumnos que muestran bajo rendimiento, entendido en este contexto como una baja actividad que el alumno desarrolla dentro del ámbito educativo formal y no solamente considerado como la obtención de calificaciones escolares, que sería el criterio social o legal.

Dichos programas tienen su base en hallazgos recientes (Román \& Hernández, 2009; Bausela, 2009), los cuales indican que existen variables ambientales, entre las cuales se puede mencionar actividades deportivas, la situación socioeconómica, tiempo con los amigos, influencia de los pares, que mediatizan los efectos del estrés, que como ya se mencionó es producido entre otras cosas por un bajo rendimiento escolar. En este sentido los programas centran su atención en objetivos como elevar la autoestima, búsqueda de soporte social y lograr una mejora en el tipo de estrategias de afrontamiento hacia los problemas (Cohen, 1997).

Dado lo anterior, se empezó a contemplar que las relaciones con los iguales contribuyen tanto al propio desarrollo cognitivo como a la socialización (Serrano y González-Herrero, 1997), ya que el soporte social es un concepto multidimensional que incluye el apoyo recibido (informativo, emocional e instrumental) y las fuentes de soporte (amigos, familia, maestros, etcétera). Es así como en los programas de apoyo es contemplada la capacidad de relacionarse con los otros, basada en el sentimiento de ser comprendido por los demás y comprender a los demás, con la meta de apoyarse juntos para incorporarse a un situación académica regular.

Por otra parte, las estrategias de afrontamiento se refieren a los esfuerzos cognitivos y conductuales para tolerar, minimizar o escapar de los efectos del estrés (Lazarus \& Folkman, 1984). Estas estrategias pueden estar centradas en la solución del problema y consistir en hacer algo para cambiar la situación estresante, o bien pueden enfocarse en la emoción, donde se persigue reducir el malestar psicológico mediante la simple evitación del estímulo nocivo. De estas dos, 
la primera es funcional mientras que la segunda es disfuncional para afrontar una condición académica irregular.

De lo anterior se concluye que todo programa de ayuda a alumnos con bajo rendimiento escolar debe contemplar el fortalecimiento de la autoestima y el autoconocimiento, la ampliación de la red de soporte social y el uso de estrategias funcionales de afrontamiento, y no sólo darle peso al aspecto académico. Es indudable que estos requisitos sólo se pueden llevar a cabo en una experiencia de grupo, con la finalidad de que sean los otros un punto de referencia que pueda contribuir en la experiencia personal de cada uno de los integrantes. Al respecto Guinion y Foulos (1980) reportan que después de una experiencia grupal, los estudiantes del primer año de universidad investigados mostraron cambios positivos en cuanto a existencialismo (flexibilidad al aplicar valores y principios propios), espontaneidad (habilidad para expresar sentimientos a través de conductas), autoaceptación (aceptación de las debilidades propias) y aceptación de la agresividad (se acepta como natural el enojo), así como en la capacidad de contactos íntimos (habilidad de desarrollar relaciones cercanas con otros).

La literatura reporta varios programas aplicados a los casos de bajo rendimiento escolar y la evaluación que se hizo de ellos; ejemplo de éstos es lo reportado por Haney y Durlank (1998), quienes realizaron un meta-análisis de 116 programas y encontraron que aquellos dirigidos a promover la autoestima fueron más significativos respecto a los cambios observados en la conducta, personalidad y funcionamiento escolar, que aquellos enfocados a otras áreas que incluían los hábitos de estudio y las habilidades sociales.

Con base en el sustento teórico planteado hasta aquí y con las experiencias académicas previas de los autores de esta investigación, el presente trabajo pretende mostrar los cambios en la tendencia actualizante de los alumnos de bajo rendimiento académico que fueron sometidos a un programa de apoyo centrado en la búsqueda de autoconocimiento y en el fortalecimiento de la autoestima.

\section{MÉTODO Participantes}

El estudio se integró con 40 alumnos del sexo masculino (20 del semestre de otoño y 20 del semestre de primavera), con edades comprendidas entre 18 y 19 años, estudiantes de las carreras de Ingeniería y Administración, candidatos a ser dados de baja de la institución por bajo desempeño académico.

\section{Instrumento}

Se utilizó el Cuestionario de Orientación Personal, elaborado por Everett Shostrom (1963) que consta de 147 reactivos dicotómicos donde los sujetos marcan la respuesta que mejor se ajusta a su manera de pensar y sentir. La validez reportada por jueces indica que existe una diferencia marcada entre autoactualizantes y no actualizantes respecto a los puntajes T. En cuanto a la validez concurrente, el Cuestionario de Orientación Personal muestra diferencias estadísticamente significativas respecto al MMPI en las escalas de depresión, desviación psicopática, psicastenia, esquizofrenia $(\mathrm{p}=.01)$ y en las de hipocondriasis, histeria y paranoia $(\mathrm{p}=.05)$.

El cuestionario consta de dos escalas de sinceridad que se denominan Tc y Ti, respectivamente. Además de las de sinceridad, el cuestionario se integra por 10 escalas clínicas, descritas a continuación: Sav (Valores de autoactualización, que hace referencia a la aceptación de los valores de otros); Ex (Existencialismo, definida como el grado de flexibilidad al aplicar principios y valores a la vida propia); Fr (Sensibilidad, indicada como sensibilidad a las necesidades y sentimientos propios); S (Espontaneidad, que es la habilidad para expresar sentimientos y llevar a cabo acciones espontáneas); $\mathrm{Sr}$ (Autoestima, que es la habilidad de reconocerse y agradarse a sí mismo); Sa (Autoaceptación, que es la capacidad de aceptar las propias debilidades); $\mathrm{Nc}$ (Construción de la naturaleza humana, que indica que la persona se ve como esencialmente buena y con capacidad de diferenciar entre polos opuestos: buenomalo, por ejemplo); Sy (Sinergia, que es la visión de las dificultades de vida como retos); A (Aceptación de la agresión, denota la capacidad de aceptar que la ira y el enojo son naturales en el ser humano); y, C (Capacidad de contacto íntimo, que es la habilidad para establecer y mantener relaciones importantes y cercanas con otros seres humanos). 
PROGRAMA DE ATENCIÓN ACADÉMICA

Consistió en sesiones grupales de ejercicios vivenciales donde se promovía el autoconocimiento y el fortalecimiento de la autoestima. Se conformó de 15 sesiones al semestre, de dos horas de duración cada una, una vez a la semana, que sumaron un total de 30 horas de trabajo. Los recursos utilizados para el trabajo de cada sesión fueron diversos, desde hojas de rotafolio, colores, revistas para recortar, cartulinas, cuestionarios, etcétera. En cada ejercicio la primera parte fue trabajo individual y la segunda consistió en socializar para compartir las experiencias.

\section{Procedimiento}

En una primera sesión se aplicó el Cuestionario de Orientación Personal. Posteriormente se llevaron a cabo las sesiones del programa de apoyo académico; en la última sesión grupal se aplicó de nuevo el Cuestionario de Orientación Personal y después se hizo la devolución a los alumnos participantes, indicándoles a cada uno las áreas que fortaleció y aquellas que debería seguir desarrollando.

\section{RESULTADOS}

Como se puede observar en la Tabla 1, el primer grupo tuvo cambios positivos en las escalas de sinceridad, además de que se observaron transformaciones significativas en dos de las 10 escalas clínicas (Construcción de la Naturaleza Humana y Aceptación de la Agresión), mientras las de Espontaneidad, Autoaceptación, Autoestima y Capacidad de Contacto Íntimo, alcanzaron la normalidad clínica estadística medidas mediante las calificaciones $\mathrm{T}$ propias de las normas de calificación de la prueba.

Tabla 1. Cambios en las puntuaciones en el test de tendencia actualizante en los alumnos del semestre de otoño

\begin{tabular}{|c|c|c|}
\hline ESCALAS & PUNTUACIÓN T & $\begin{array}{c}\text { PUNTUACIÓN T } \\
\text { SEGUNDA APLICACIÓN }\end{array}$ \\
\hline TC & 44 & 47 \\
\hline TI & 48 & 49 \\
\hline Sav & 26 & 26 \\
\hline Ex & 45 & 45 \\
\hline Fr & 46 & 46 \\
\hline S & 48 & 51 \\
\hline Sr & 50 & 54 \\
\hline Sa & 52 & 58 \\
\hline Nc & 58 & 48 \\
\hline Sy & 39 & 39 \\
\hline A & 42 & 46 \\
\hline C & 48 & 51 \\
\hline
\end{tabular}

Sav = Valores de autoactualización: aceptación de los valores de otros

$\mathrm{Ex}=$ Existencialismo: Flexibilidad de aplicar principios y valores a la propia vida

$\mathrm{Fr}=$ Sensibilidad: Sensibilidad a las necesidades y sentimientos propios

$\mathrm{S}=$ Espontaneidad: Habilidad para expresar sentimientos y llevar a cabo acciones espontáneas

$\mathrm{Sr}=$ Autoestima: Habilidad de reconocerse y agradarse a sí mismo

$\mathrm{SA}=$ Autoaceptación: Capacidad de aceptar las propias debilidades

$\mathrm{Nc}=$ Construcción de la naturaleza humana: La persona se vé como esencialmente buena y tiene la capacidad de diferenciar entre polos opuestos

Sy $=$ Sinergia: Visión de las dificultades de la vida como retos

$A=$ Aceptación de la agresión: Se acepta la ira y el coraje como naturales en el ser humano

$\mathrm{C}=$ Capacidad de contacto íntimo: Habilidad para establecer y mantener relaciones importantes y cercanas con otros seres humanos 
En cuanto al segundo grupo también se observaron cambios positivos en las escalas de sinceridad, además de en cuatro de las 10 escalas clínicas (Valores de Actualización, Sensibilidad, Construcción de la Naturaleza Humana y Aceptación de la Agresión) y otras cuatro alcanzaron la normalidad estadística (Espontaneidad, Autoaceptación, Autoestima y Capacidad de Contacto Íntimo). (Ver Tabla 2).

\section{DISCUSIÓN}

Los resultados del presente estudio van más allá de lo encontrado por Hay, et al., (1998) y Hartocollis (1999), ya que no sólo se detectó un autoconcepto negativo y una baja autoestima en los alumnos con bajo rendimiento escolar antes de iniciar el programa, sino que en general se observaron problemas en la aceptación de valores de los otros, baja sensibilidad a las necesidades y sentimientos propios, poca espontaneidad, dificultad para ver los problemas como retos, baja tolerancia a la ira y el enojo, además de poca capacidad de contacto íntimo.

Los hallazgos, en este sentido, indican que el rendimiento escolar se ve afectado por factores personales, entre ellos la autoestima y el autoconocimiento, mismos que propician en los alumnos la motivación a desempeñarse de mejor manera en el ámbito escolar,

Tabla 2. Cambios en las puntuaciones en el test de tendencia actualizante en los alumnos del semestre de primavera

\begin{tabular}{|c|c|c|}
\hline ESCALAS & $\begin{array}{c}\text { PUNTUACIÓN T } \\
\text { PRIMERA APLICACIÒN }\end{array}$ & $\begin{array}{c}\text { PUNTUACIÓN T } \\
\text { SEGUNDA APLICACIÒN }\end{array}$ \\
\hline Tc & 44 & 47 \\
\hline TI & 47 & 48 \\
\hline Sav & 24 & 26 \\
\hline Ex & 37 & 37 \\
\hline $\mathrm{Fr}$ & 32 & 34 \\
\hline$S$ & 49 & 51 \\
\hline $\mathrm{Sr}$ & 52 & 56 \\
\hline $\mathrm{Sa}$ & 52 & 58 \\
\hline Nc & 40 & 48 \\
\hline Sy & 38 & 38 \\
\hline$A$ & 43 & 47 \\
\hline C & 50 & 52 \\
\hline
\end{tabular}

Sav $=$ Valores de autoactualización: eceptación de los valores de otros

$\mathrm{Ex}=$ Existencialismo: Flexibilidad de aplicar principios y valores a la propia vida

$\mathrm{Fr}=$ Sensibilidad: Sensibilidad a las necesidades y sentimientos propios

$\mathrm{S}=$ Espontaneidad: Habilidad para expresar sentimientos y llevar a cabo acciones espontáneas

$\mathrm{Sr}=$ Autoestima: Habilidad de reconocerse y agradarse a sí mismo

$\mathrm{SA}=$ Autoaceptación: Capacidad de aceptar las propias debilidades

$\mathrm{Nc}=$ Construcción de la naturaleza humana: La persona se vé como esencialmente buena y tiene la capacidad de diferenciar entre polos opuestos

Sy= Sinergia: Visión de las dificultades de la vida como retos

$\mathrm{A}=$ Aceptación de la agresión: Se acepta la ira y el coraje como naturales en el ser humano

$\mathrm{C}=$ Capacidad de contacto íntimo: Habilidad para establecer y mantener relaciones importantes y cercanas con otros seres humanos 
no se sabe si son la causa o la consecuencia del desempeño, ya que si la autoestima es contingente al éxito, los estudiantes pueden percibir el fracaso o la crítica como censura a su valía y sentirse incapaces de desempeñarse mejor, no obstante dan directrices de hacia dónde podrían orientarse las medidas de intervención o de prevención del rendimiento bajo, ya que no bastaría el trabajo con variables como los hábitos de estudio, las habilidades mentales o el entrenamiento en habilidades como autoeficacia escolar, por ejemplo, sino que tendrían que encaminarse hacia aspectos personales, familiares y sociales en el tratamiento de este fenómeno, donde las interacciones personales tanto a nivel profesor/alumno, entre compañeros, familiares y amigos, fortalece estos factores.

Lo anterior se fundamenta en el estudio realizado por Haney y Durlank (1998), en el sentido de que los programas orientados al fortalecimiento de la autoestima logran resultados significativos satisfactorios en el funcionamiento escolar.

Además de que se observaron cambios en las escalas del instrumento aplicado en este trabajo, entre una y otra evaluación, se encontró que de los 40 alumnos participantes, 38 lograron volver a su estatus académico regular, al incrementar sus calificaciones en el trascurso del semestre en el que cursaron el programa de atención y en el posterior, con lo que pudieron superar el riesgo de ser dados de baja y sólo dos de los casos evaluados fueron dados de baja por no alcanzar los estándares de calificación requeridos por la institución educativa en la que estudiaban.

La idea planteada por algunos autores (Dubois, Bull, Sherman \& Roberts, 1998), de que los altos niveles de autoestima ayudan a incrementar mejores niveles de desarrollo académico, se ve apoyada con los hallazgos en este estudio, ya que se observa que los alumnos incrementaron sus niveles de autoestima, a la vez que mejoraron su rendimiento escolar, y por consiguiente su autoconcepto se elevó, ya que conjuntamente con los factores internos que se controlaron, lograron en el alumno una actitud positiva ante las actividades académicas.

Así que las características del programa remedial al que fueron sometidos los alumnos, que se centró en el autoconocimiento y en el fortalecimiento de la autoestima, tuvo un impacto positivo, ya que les ayudó a desarrollar herramientas para poder hacer frente al estrés, a buscar estrategias de afrontamiento adecuadas y a hacer una exploración de su esfera personal. Lo anterior permite corroborar lo dicho por Cohen (1997), quien indicó que el estrés está presente en personas con bajo rendimiento escolar y se convierte en una fuente de dificultades para la persona que lo experimenta, ya que no le permite avanzar en los diferentes ámbitos personales en los que se desarrolle. Además, cabe resaltar que de acuerdo con lo planteado por el modelo de Maslow (DiCaprio, 1989), las necesidades de autoactualización son únicas y cambiantes, dependiendo del individuo y de sus circunstancias, pero están ligadas con la necesidad de satisfacer la naturaleza individual y con el cumplimiento del potencial de crecimiento y que uno de los medios para satisfacer esta necesidad de autoactualización es a través de la realización de actividades laborales o vocacionales, además de realizarlas con resultados favorables y que en esta etapa de vida una de las tareas más importantes de estos jóvenes está centrada en las actividades y los logros escolares, pero si son deficientes, esto impacta de forma negativa otras áreas de su vida, y por el contrario, una mejora en el conocimiento del sí mismo y una clarificación en esta área personal, tiene efectos en las esferas escolares, tal y como se refleja en los resultados del programa aplicado.

Para apoyar los resultados referentes a la autoestima, misma que observa cambios de una aplicación a otra en esta investigación, González y Touron (1992; como se citó en Gázquez, Pérez, Ruíz, Miras \& Vicente, 2006), indican que la autoestima es la parte valorativa del autoconcepto que da un modo de orientación hacia sí mismo y que atribuye un valor a la persona; que cuando ésta se siente autocompetente se produce un incremento en su implicación activa en el proceso de aprendizaje, ya que esto se traduce en mayor nivel de esfuerzo, más persistencia ante las dificultades, mayor motivación intrínseca y orientación hacia la consecución de metas (Zimmerman, Bandura \& Martínez-Pons, 1992; como se citó en Gázquez, et al., 2006), además de que se apunta una relación bidireccional entre la autoestima y el rendimiento académico, pues lo reportado por Aunola, Stattin \& Nurmi, 2000 (como se citó en Gázquez, et al., 2006) apunta a que cuando las personas presentan problemas o dificultades en sus 
aprendizajes también poseerán déficits en su autovaloración.

Es así que para García (1982), (como se citó en Gázquez, et al., 2006), la carencia de estima propia aparece sistemáticamente relacionada con ansiedad, depresión, sensación de fracaso, dificultades en la selección de estrategias de afrontamiento al estrés y una la no aceptación de la imagen personal, que entre otros factores, pueden inhibir o entorpecer las tareas relacionadas con la actividad académica y el reverso de la moneda es que el logro académico puede verse afectado de manera positiva al trabajar directamente con la autoestima.

En este sentido, González-Pienda, Núñez-Pérez, González-Pumariega y García-García (1997), apoyan la hipótesis de la vertiente personal está contenida en variables de desempeño académico, sumando así tres ámbitos de análisis: la cognición, la conación y el afecto. En el ámbito cognitivo se incluyen dos tipos de variables: las habilidades y los conocimientos previos; el ámbito conativo está integrado por aquellas características de la persona que con el paso del tiempo se conceptualizan como estilos propios de enfrentarse a las tareas de aprendizaje (de los que se reconocen dos: menos dependientes de la tarea específica y más relacionados con el ámbito de las habilidades cognitivas) y los estilos de aprendizaje (menos relacionados con el ámbito cognitivo y más vinculados al ámbito del comportamiento y de las tareas de aprendizaje); en tercer lugar, el ámbito afectivo se encuentran dos variables importantes para el aprendizaje escolar: por una parte,

\section{REFERENCIAS}

Cohen, F. (1997). Measurement of coping. New York: Wiley.

DiCaprio, N. S. (1989). Teorías de la personalidad. México: McGraw Hill.

Dubois, D. (1992). A prospective study of life stress, social support and adaptation in early adolescence. Child Development, 6, 542-557.

Dubois, D., Bull, C. Sherman, M. \& Roberts, M. (1998). Self-esteem and adjusment in early adolescence: A social-contextual perspective. Journal of youth and adolescence, 25, 557-583. constructos como la ansiedad y el autoconcepto y por otra, la motivación académica. De tal forma que en el ámbito del ambiente de aprendizaje escolar son tres las variables que en su conjunto permiten predecir el grado de éxito o fracaso en la tarea: 1) el poder: inteligencia y aptitudes; 2) el querer, y 3 ) el modo de ser (Hernández, 1991; citado por González-Pienda, et al., 1997), de tal forma que esta investigación reporta un área de trabajo personal y en el desarrollo de estrategias de afrontamiento a tareas y a problemas, que de no atenderse, incrementan la problemática reflejada en el ámbito escolar.

Para concluir, los resultados de la presente investigación apoyan los hallazgos de Pope (1988), Dumont y Provost (1999), en lo referente a que la confianza en sí mismo y en las habilidades personales e intelectuales en general, contribuyen en lo académico, que sería el equivalente de un buen nivel de bienestar psicológico, son buenos predictores del desarrollo académico exitoso, se reflejan en buenos niveles de dedicación, de autoeficacia académica y de satisfacción consigo mismo y con los estudios, así como con bajos niveles de propensión al abandono (Salanova-Soloria, Martínez-Martínez, Bresó-Esteve, Llorens-Gumbau \& Grau-Gumbau, 2005), además de que hay una estrecha relación entre la experimentación de ansiedad o de estrés con el desempeño académico (HernándezPozo, Coronado-Álvarez, Araujo-Contreras \& CerezoReséndiz, 2008), por lo que la sugerencia es centrar más la atención en variables personales que impacten en el ámbito académico.

Dumont, M.y Provost, M. (1999). Resilience in adolescents: Protective role of social support, coping strategies, self-esteem and social activities on experience of stress and depression. Journal of youth and adolescence, 28, 343-363.

Gázquez, J. J., Pérez, M. C., Ruíz, M. I., Miras, F. \& Vicente, F. (2006). Estrategias de aprendizaje en estudiantes de enseñanza secundaria obligatoria y su relación con la autoestima. International journal of Psychology and Psychological Therapy. April, 6 (001), 51-62. 
González-Pienda, J. A., Núñez-Pérez, C., González-Pumariega, S. y García-García, M. S. (1997). Autoconcepto, autoestima y aprendizaje escolar. Psicothema. 9 (2), 272-289.

Guinion, R. y Foulos, F. (1980). Caring and Competence. Ethics and Regulation, 4 (2), 36-43.

Hartocollis, P. (1999). Quality of depressive experiences in borderline personality disorders: Differences between patients with borderline personality disorder and patients with higher levels of personality organization. Bulletin of the Meninger Clinic, 68, 9-22.

Hernández-Pozo, M. R., Coronado-Álvarez, O. AraujoContreras, V. y Cerezo-Reséndiz, S. (2008). Desempeño académico de universitarios en relación con ansiedad escolar y autoevaluación. Acta colombiana de psicología, 11, (1), 13-23.

Filozof, E., Albertin, H. Jones, C. \& Steme, S. (1998). Relationship of adolescent self-steem to selected academic variables. The journal of school health, 68, 68-72.

Haney, P. y Durlank, J. (1998). Changing self-steem in children and adolescents: A metanalitic review. Journal of clinical child psychology, 27, 423-433.

Hay, I., Ashman, A., \& Van Kraayenoord, C. (1998). Educational characteristics of students with high or low self-concept. Psychology in the schools, Oct, 55-67.
Lafarga, J., \& Gómez, J. (1989). Desarrollo del potencial humano, Vol. 3. México: Trillas.

Lazarus, R. \& Folkman, S. (1984). Stress appraisal and coping. New York: Springer Publishing Company.

Maslow, A. (1954). Motivation and personality. Nueva York: Harper \& Row.

Pope, A. (1988). Self-esteem enchacement with children and adolescents. New York: Pergamon Press.

Román, C. \& Hernández, Y. (2009). Variables psicosociales y su relación con el desempeño académico de estudiantes de primer año de la Escuela Latinoamericana de Medicina. Revista Iberoamericana de Educación, 10, 1-9.

Salanova-Soloria, M.; Martínez-Martínez, I. A., BresóEsteve, E., Llorens-Gumbau, S. \& Grau-Gumbau, R. (2005). Bienestar psicológico en estudiantes universitarios: Facilitadores y obstaculizadores del desempeño académico. Anales de psicología. Junio, 21 (001), 170-180.

Serrano, J. y González-Herrero, M. (1997). Aprendizaje cooperativo en matemáticas. España: Secretariado de Publicaciones de la Universidad de Murcia.

Shostrom, E. (1963). Personal Orientation Inventory. USA: Educational and Industrial Testing Service. 